\title{
Extranodal marginal zone lymphoma: an unusual cause of gastric subepithelial tumor
}

\author{
Eun Young Park and Gwang Ha Kim
}

Department of Internal Medicine, Pusan National University School of Medicine and Biomedical Research Institute, Pusan National University Hospital, Busan, Korea

\section{Received: February 16, 2020 \\ Revised : March 11, 2020 \\ Accepted: March 12, 2020 \\ Correspondence to \\ Gwang Ha Kim, M.D. \\ Tel: +82-51-240-7869 \\ Fax: +82-51-244-8180 \\ E-mail: doco224@pusan.ac.kr https://orcid.org/0000-0001- 9721-5734}

A 54-year-old man presented to our department for evaluation of a subepithelial tumor (SET) in the stomach, which was incidentally detected on screening endoscopy. Endoscopy demonstrated a solid, round SET covered with normal gastric mucosa, measuring approximately $20 \mathrm{~mm}$ in diameter located on the posterior wall of the gastric fundus (Fig. 1A). Endoscopic ultrasonography revealed a $22 \mathrm{~mm}$ homogenously hypoechoic mass located in the submucosal and proper muscle layers of the stomach (Fig. 1B). The size of SET increased compared to the previous endoscopy 1 year before and endoscopic ultrasound presumed the diagnosis of gastrointestinal stromal tumor. Therefore, we decided to perform endoscopic submucosal dissection for the SET owing to the possibility of it being ma- lignant; en bloc resection was achieved successfully (Fig. 1C).

Histopathological examination of the resected specimen revealed a well-delineated, sharply circumscribed, oval lymphoid nodule located in the submucosa without involvement of the mucosa (Fig. 2A). The lymphoid cells were mostly small lymphocytes with dense chromatin (Fig. 2B). Immunostaining was positive for $\mathrm{CD}_{2} \mathrm{O}$ and B-cell lymphoma 2 (bcl-2) but negative for $\mathrm{CD}_{3}, \mathrm{CD}_{5}, \mathrm{CD} 10$, bcl-6, cyclin $\mathrm{D}_{1}$, and SOX11. Based on these findings, the tumor was diagnosed as gastric extranodal marginal zone lymphoma without mucosal involvement. Tests for Helicobacter pylori, including rapid urease test, tissue biopsy, serum antibody, and urea breath test were negative. Abdominopelvic computed tomography
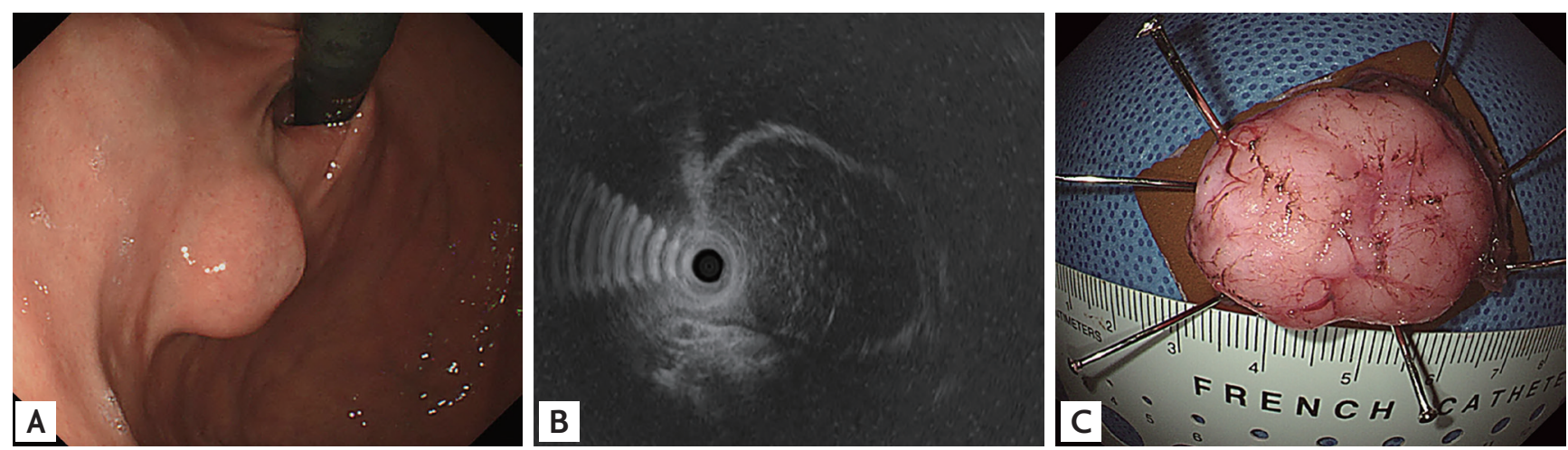

Figure 1. (A) Upper endoscopy reveals a solid, round subepithelial tumor covered with normal gastric mucosa, measuring approximately $20 \mathrm{~mm}$ in diameter located on the posterior wall of the gastric fundus. (B) On endoscopic ultrasonography, a 22 $\mathrm{mm}$ homogenously hypoechoic mass is located in the submucosal and proper muscle layers of the stomach. (C) The inner surface of the resected tumor. 

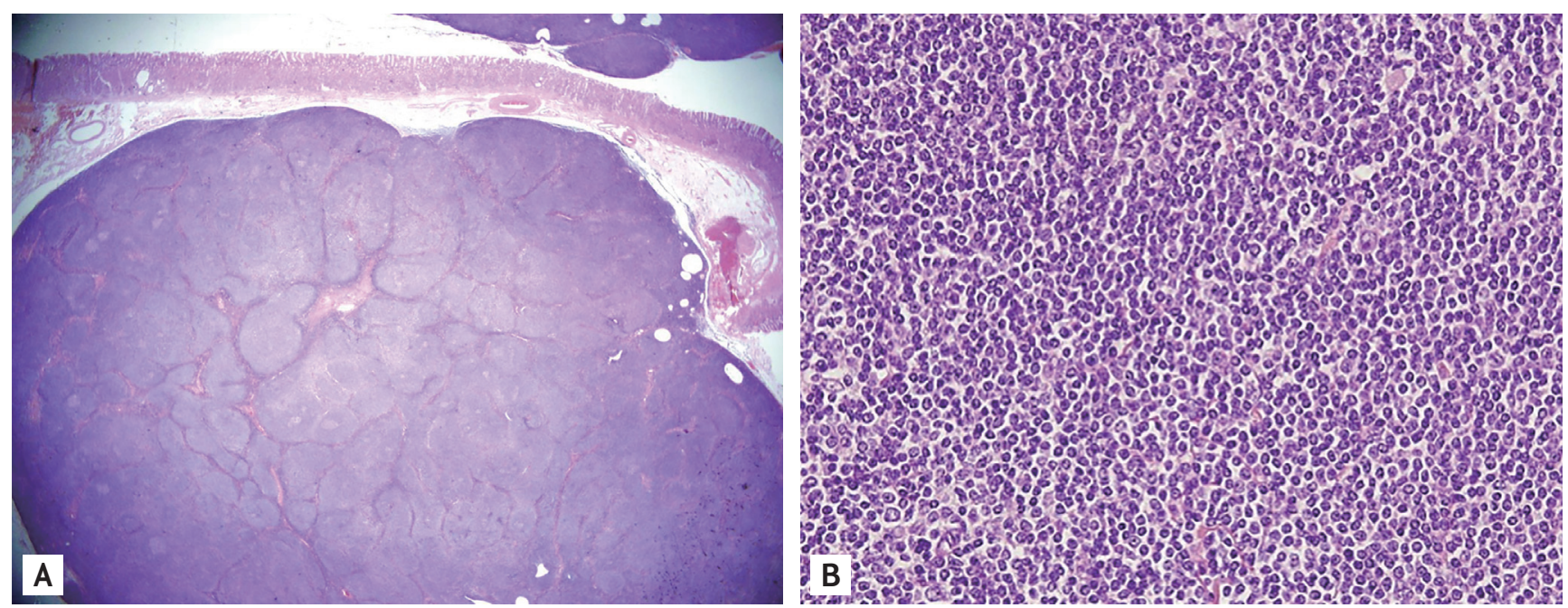

Figure 2. (A) Histopathological examination reveals a well-delineated, sharply circumscribed, oval lymphoid nodule located in the submucosa without involvement of the mucosa $(\mathrm{H} \& \mathrm{E}, \times 12.5)$. (B) The lymphoid cells are mostly small lymphocytes with dense chromatin $(\mathrm{H} \& \mathrm{E}, \times 400)$.

(CT) and positron emission tomography-CT (PET-CT) revealed perigastric lymph node enlargement. Bone narrow biopsy showed no involvement of the bone marrow by the lymphoma. The final gastric lymphoma stage was IIE1 based on the Ann Arbor clinical staging, and radiotherapy was administered for perigastric lymph node involvement. No sign of recurrence were seen at the 18-month follow-up visit.

Extranodal marginal zone lymphoma is the most common form of extralymphatic lymphoma, with the highest frequency occurring in the mucosa-associated lymphoid tissue. About 50\% to 60\% of these lymphomas occur in the gastrointestinal tract; the stomach is the most common site. Gastric extranodal marginal zone lymphomas progress very slowly, rarely metastasize systemically, and in many cases complete remission can be achieved by eradication of $H$. pylori alone. When extranodal marginal zone lymphomas are multiple and metastasize to lymph nodes, radiotherapy, or systemic chemotherapy are often performed.

All authors declare that informed consent was obtained from the patient to publish these images.

\section{Conflict of interest}

No potential conflict of interest relevant to this article was reported. 